\title{
PREVALENCIA DE EMBARAZO EN ADOLESCENTES, YOPAL-CASANARE, COLOMBIA, 2013-2014
}

\section{Prevalence of teenage pregnancy in Yopal-Casanare, Colombia, 2013-2014}

\author{
Alejandra Hernández-Ávila ${ }^{1}$; Edwin Jesús Barrera-Rodríguez, $M^{2}$; \\ Oneida Castañeda-Porras, $\mathbf{M S c}^{3}$
}

Recibido: febrero 10/17 - Aceptado: agosto 23/17

\section{RESUMEN}

Objetivo: establecer la prevalencia de embarazo en mujeres adolescentes desde los registros de nacidos vivos del municipio de Yopal entre 2013-2014.

Materiales y métodos: estudio descriptivo transversal desde la base de datos del módulo de nacidos vivos del Registro Único de Afiliados del Departamento Nacional de Estadística (DANE), suministrado por la Secretaría de Salud de Yopal. Se incluyeron registros completos de toda gestante menor de 19 años. Se estudiaron sus características sociodemográficas, antecedentes de la gestación y características específicas del nacimiento. Análisis mediante estadística descriptiva.

Resultados: el 21,9\% (1.286/5.848) de registros correspondieron a gestantes adolescentes entre 10 19 años, con edad promedio 17,4 \pm 1,47 años; edad promedio del padre 23,9 \pm 5,66 años; mediana de embarazos $=1$ (rango $=1-4)$; más de cuatro

1 Enfermera; Especialista en Gerencia en Salud y Epidemiología, Universidad de Boyacá, Programa de Especialización en Epidemiología, Secretaría de Salud de Casanare (Colombia).adaher03@hotmail.com

2 Médico; Especialista en Auditoría en Salud, Salud Ocupacional y Epidemiología, Universidad de Boyacá, Programa de Especialización en Epidemiología, Secretaría de Salud Municipal de Yopal, Casanare (Colombia). edwinsaludyopal@hotmail.com

3 Psicóloga; Magíster en Salud Pública; Epidemióloga de Campo, Universidad de Boyacá; Programa de Especialización en Epidemiología. Grupo de Investigación SMC-AS Unidad de Investigaciones. Bogotá (Colombia). ocastaneda@smc-as.com controles prenatales 707 (55,0\%); primigestantes 1.033 (80,3\%); periodo intergenésico menor de dos años 54 (25,7\%); parto espontáneo 894 (69,5\%) y cesárea 392 (30,5\%); presentaron bajo peso al nacer $54(4,2 \%)$ neonatos.

Conclusión: la prevalencia del embarazo en adolescentes en Yopal fue superior a la nacional, de 19,5\%; las gestantes adolescentes del área rural acceden menos a los controles prenatales. Estos datos conllevan revisar y fortalecer las estrategias municipales de prevención de este evento.

Palabras clave: embarazo en adolescencia, estadísticas vitales, nacimiento vivo, atención prenatal

\section{ABSTRACT}

Objective: To determine the prevalence of teenage pregnancy on the basis of live birth records in the Municipality of Yopal between 2013-2014.

Materials and methods: Descriptive cross-sectional study conducted on the basis of the live birth module database contained in the Single Registry of the National Statistics Department (DANE), made available by the Health Secretariat of Yopal. Full records of all pregnant women under 19 were included. Sociodemographic characteristics, pregnancy history and specific characteristics of the birth were studied. Descriptive statistical analysis was done.

Results: Of the records, $21.9 \%(1,286 / 5,848)$ were pregnant teens between $10-19$ years, with a mean 
age of $17.4 \pm 1.47$ years; mean age of the fathers was $23.9 \pm 5.66$ years; pregnancy median $=1$ (range $=1-4)$; more than four prenatal visits 707 (55.0\%); primigravidae 1,033 (80.3\%); interpregnancy period of less than two years 54 (25.7\%); spontaneous delivery 894 (69.5\%) and cesarean section 392 (30,5\%); low birth weight, 54 (4.2\%) neonates.

Conclusion: The prevalence of pregnancy among adolescents in Yopal was higher than the national prevalence, at $19.5 \%$; pregnant adolescents who come from the rural areas have access to a smaller number of prenatal visits. These data point to the need of revisiting and strengthening municipal strategies for the prevention of teenage pregnancy. Key words: Teenage pregnancy, vital statistics, live birth, prenatal care.

\section{INTRODUCCIÓN}

La Organización de las Naciones Unidas ha definido como adolescentes al grupo de personas entre los 10 y 19 años de edad (1). Para el 2010, se calculaba que en América Latina los adolescentes representaban una quinta parte de la población, con 110 millones de personas. Esta es una edad que conlleva riesgos para la salud general por un alto consumo de bebidas azucaradas, pobre higiene dental, uso de bebidas alcohólicas y drogas, comportamientos sexuales de riesgo como: el no uso de condón, no uso de métodos de planificación y alta tasa de embarazos no deseados (2).

El embarazo adolescente es motivo de preocupación para la salud pública a nivel global ya que se acompaña de una importante morbilidad derivada de factores biológicos, socioculturales y económicos que comprometen el binomio madre adolescentehijo (3-5). Por ejemplo, es frecuente el bajo peso materno y bajo peso fetal y el parto pretérmino $(5,6)$, una pobre asistencia al control prenatal y baja tamización para el VIH y la sífilis en la gestación; desde el punto de vista económico afecta la posibilidad de estudiar, por lo que usualmente las madres adolescentes se ven relegadas a trabajos con bajos salarios, lo que perpetúa la pobreza (1, 4). Este problema tiene repercusiones que afectan desde lo individual, pasando por lo familiar, hasta la sociedad en su conjunto (7-9). La Organización Mundial de la Salud (OMS) estimó que para el 2014 había alrededor de 16 millones de adolescentes embarazadas entre 15 y 19 años, y 1 millón de niñas gestantes menores de 15 años daban a luz cada año, la mayoría en los países de ingresos bajos y medios (7). La tasa de fecundidad de las adolescentes en el periodo 2005-2010 para la región de las Américas fue de 66\%o en mujeres entre 15 y 19 años, inferior a la de África (115\%), y superior a la de Asia Sudoriental (49\%o), Europa (23\%o), Mediterráneo Oriental (37\%) y Pacífico Occidental (13\%o) (8). En 2014, el Fondo de Población de las Naciones Unidas (UNFPA) estimó la prevalencia de embarazo adolescente para algunos países de la región así: Paraguay, 11,6\%; Perú, 13,2\%; Ecuador, 19,4\%; Brasil, 22,3\%; El Salvador, 22,8\% y Guatemala, $21,8 \%(10)$.

En Colombia, la tasa de fertilidad para el mismo periodo fue de $91 \%$; en el grupo etario de 10-14 años pasó de $3 \%$ en 1998 a 4\%o en 2009, y en el de 15-19 años pasó de 99 a $92 \%$ (11), mientras que en 2010 fue de 84\%o. La prevalencia de embarazo adolescente pasó de 20,5\% en 2005 a 19,5\% en 2010 (12), muy por encima de la meta establecida para el 2015 de mantener el límite por debajo del $15 \%$ (13), meta ratificada para el $2021(14,15)$. Un estudio demográfico estimó que en el departamento de Casanare, en la Orinoquia colombiana, la tasa de fertilidad en las niñas de 10-14 años pasó de 5,0\%o en 1998 a 4,1\%o en 2009; y en las adolescentes de 15-19 años cambió de 125,6\%o en 1998 a 85,5\%o en 2009 (11), y a $130 \%$ en 2010, año en el que la proporción de adolescentes alguna vez embarazadas fue de $26 \%$ (12). Ahora, según las estimaciones del Departamento Nacional de Estadística (DANE), para el 2012, de acuerdo con el número de nacimientos según el municipio de residencia de la madre, Yopal, capital departamental, ocupó el primer lugar de adolescentes embarazadas con el 22,4\%, tres 
puntos porcentuales por encima de la media nacional (19,5 \%) (13). Por esta razón ese mismo año, con el propósito de implementar la estrategia para prevenir el embarazo en la adolescencia y promover proyectos de vida para los niños, niñas y adolescentes (Consejo Nacional de Política Económica y Social - Conpes Social 147), el Departamento Nacional de Planeación (DNP) incluyó el municipio de Yopal entre los 185 municipios del país priorizados (16).

No se conoce el impacto de esta estrategia en Yopal, pero es importante determinar la razón de prevalencia de embarazo en adolescentes como medición del primer y segundo año del lanzamiento de la misma. Por tanto, el objetivo de este estudio fue determinar la prevalencia del embarazo en adolescentes en el municipio de Yopal (Casanare, Colombia), para el periodo comprendido entre el 2013 y 2014.

\section{MATERIALES Y MÉTODOS}

Se llevó a cabo un estudio descriptivo de corte trasversal. Se incluyeron las gestantes con edades entre 10 y 19 años de edad, que contaban con registro completo del Registro Único de Afiliados de nacimientos y defunciones (RUAF-ND), y con lugar de residencia en el municipio de Yopal para el periodo del 01 de enero de 2013 al 31 de diciembre de 2014. Se excluyeron aquellos registros en los que en alguna de las casillas se encontrase diligenciado el campo como "sin información", "ignorado" o estuviesen vacías. La muestra estuvo constituida por todos los registros de gestantes.

Procedimiento. Para acceder a la información se realizó el trámite correspondiente y solicitud ante la Secretaría de Salud Municipal de Yopal, responsable de consolidar la información reportada por las entidades encargadas de la atención del parto y el diligenciamiento del certificado de nacido vivo. Posteriormente, accedimos a la base de datos de nacidos vivos del RUAF-ND, disponible como hoja de cálculo en Microsoft Office ${ }^{\circledR}$ Excel, que contiene la información de la edad de las gestantes y de los recién nacidos vivos, y las otras variables medidas.
A continuación, se llevó a cabo el proceso de depuración de la información de todas y cada una de las variables de estudio para su posterior análisis.

Variables de estudio. Características sociodemográficas de la madre (edad, escolaridad, estado civil, área de procedencia, tipo de régimen de afiliación al Sistema General de Seguridad Social en Salud - SGSSS) y edad del padre; antecedentes de la gestación (control prenatal, número de embarazos y periodo intergenésico); y características específicas del nacimiento (tipo de parto, multiplicidad de embarazos, tiempo de gestación, peso al nacer y sexo del recién nacido).

El análisis de la información se realizó mediante el uso de estadística descriptiva, con medidas de frecuencia, tendencia central y dispersión. Se presenta la razón de prevalencia de gestación en adolescentes. Se utilizó el programa estadístico SPSS versión $22 ®$.

Aspectos éticos. El estudio fue aprobado por el Comité de Investigaciones y Currículo de la Universidad de Boyacá, y contó con la autorización de la Secretaría de Salud de Yopal para tener acceso a la información registrada en el módulo de estadísticas vitales RUAF-ND.

\section{RESULTADOS}

La base de datos suministrada estaba constituida por 6.077 registros de nacidos vivos; una vez aplicados los criterios de inclusión se totalizaron 5.848 (96,2 \%) recién nacidos vivos: 2.846 para el 2013 y 3.002 para el 2014, de los cuales 1.286 (21,9\%) correspondían a embarazos en mujeres de 10 a 19 años, un total de 628 en 2013 y 658 en 2014. La razón de prevalencia en el 2013 fue de 22,1 \% y en 2014 de 21,9\%.

La edad promedio de la madre fue de 17,4 \pm 1,5 años; por grupos etarios se distribuyó así: 1014 años, 48 (3,7\%); 15-19 años, 1.238 (96,3\%). La edad promedio del padre fue de 23,9 \pm 5,7 años; agrupados según la edad: 10-19 años, 233 (18,9\%); 20-29 años, 887 (69,0\%); 30-60 años, 166 (12,9\%).

La población evaluada pertenecía principalmente al área urbana, cursaba educación secun- 
daria, estaba en unión libre y afiliada al régimen de seguridad social subsidiado por el Estado (tabla 1). Respecto a los antecedentes de la gestación, el $42 \%$ de las adolescentes asistieron a menos de 5 controles prenatales, hasta una quinta parte cursaba su segundo embarazo, y entre estas últimas, en una cuarta parte el periodo intergenésico fue menor de dos años (tabla 2).

En cuanto a las características específicas del nacimiento, hasta un 30\% fue parto por cesárea y hubo una baja frecuencia de bajo peso al nacer o de macrosomía (tabla 2).

\section{DISCUSIÓN}

La prevalencia de periodo del embarazo adolescente en Yopal fue similar en los dos años estudiados, con un mayor valor para el 2013. La edad promedio materna fue de 17,4 años, con predominio del grupo de 15 a 19 años (17-19). Cerca de la mitad de las adolescentes gestantes tuvo menos de cuatro controles

\begin{tabular}{|c|c|c|c|c|c|c|c|c|c|c|c|c|}
\hline \multirow{2}{*}{ Variable } & \multicolumn{6}{|c|}{2013} & \multicolumn{6}{|c|}{2014} \\
\hline & $10-14$ & $\%$ & $15-19$ & $\%$ & Total & $\%$ & $10-14$ & $\%$ & $15-19$ & $\%$ & Total & $\%$ \\
\hline \multicolumn{13}{|c|}{ Lugar de procedencia } \\
\hline Rural & 5 & 26 & 102 & 17 & 107 & 17 & 4 & 14 & 122 & 19 & 126 & 19 \\
\hline Urbano & 14 & 74 & 507 & 83 & 521 & 83 & 25 & 86 & 507 & 81 & 532 & 81 \\
\hline \multicolumn{13}{|c|}{ Escolaridad } \\
\hline Primaria & 11 & 58 & 108 & 18 & 119 & 19 & 11 & 38 & 100 & 16 & 111 & 17 \\
\hline Secundaria & 8 & 42 & 453 & 74 & 461 & 73 & 18 & 62 & 496 & 79 & 514 & 78 \\
\hline Técnico/Tecnológico & 0 & 0 & 30 & 4,9 & 30 & 4,8 & 0 & 0 & 21 & 3,3 & 21 & 3,2 \\
\hline Universidad & 0 & 0 & 15 & 2,5 & 15 & 2,4 & 0 & 0 & 12 & 1,9 & 12 & 1,8 \\
\hline Ninguno & 0 & 0 & 3 & 0,5 & 3 & 0,5 & 0 & 0 & 0 & 0 & 0 & 0 \\
\hline \multicolumn{13}{|c|}{ Estado civil } \\
\hline Soltera & 11 & 58 & 165 & 27 & 176 & 28 & 20 & 69 & 173 & 28 & 193 & 29 \\
\hline Casada & 0 & 0 & 14 & 2,3 & 14 & 2,2 & 0 & 0 & 11 & 1,7 & 11 & 1,7 \\
\hline Unión Libre & 8 & 42 & 427 & 70 & 435 & 69 & 9 & 31 & 444 & 71 & 453 & 69 \\
\hline Separada & 0 & 0 & 2 & 0,3 & 2 & 0,3 & 0 & 0 & 0 & 0 & 0 & 0 \\
\hline Otro & 0 & 0 & 1 & 0,2 & 1 & 0,2 & 0 & 0 & 1 & 0,2 & 1 & 0,2 \\
\hline \multicolumn{13}{|c|}{ Tipo de régimen de salud } \\
\hline Contributivo & 3 & 16 & 179 & 29 & 182 & 29 & 2 & 6,9 & 182 & 29 & 184 & 28 \\
\hline Subsidiado & 16 & 84 & 412 & 68 & 428 & 68 & 26 & 90 & 436 & 69 & 462 & 70 \\
\hline Especial & 0 & 0 & 5 & 0,8 & 5 & 0,8 & 1 & 3,4 & 7 & 1,1 & 8 & 1,2 \\
\hline No afiliado & 0 & 0 & 13 & 2,1 & 13 & 2,1 & 0 & 0 & 4 & 0,6 & 4 & 0,6 \\
\hline
\end{tabular}




\begin{tabular}{|c|c|c|}
\hline \multicolumn{3}{|c|}{$\begin{array}{c}\text { Tabla } 2 . \\
\text { Antecedentes de la gestación y } \\
\text { resultados obstétricos de las gestantes } \\
\text { adolescentes en Yopal, Colombia, 2013-2014 }\end{array}$} \\
\hline \multirow{2}{*}{ Variables } & 2013 & 2014 \\
\hline & n (\%) & n (\%) \\
\hline \multicolumn{3}{|c|}{ Control prenatal } \\
\hline 0 Ninguno & $44(7,0)$ & $37(5,6)$ \\
\hline 1-4 Controles & $258(41,1)$ & $240(36,5)$ \\
\hline$>4$ Controles & $326(51,9)$ & $381(57,9)$ \\
\hline \multicolumn{3}{|c|}{ Número de embarazos } \\
\hline Primigestante & $501(79,8)$ & $532(80,9)$ \\
\hline Multigestante & $127(20,2)$ & $126(19,1)$ \\
\hline \multicolumn{3}{|c|}{ Periodo intergenésico } \\
\hline Menor a 2 años & $29(27,4)$ & $25(24,0)$ \\
\hline 2 años y más & $77(72,6)$ & $79(76,0)$ \\
\hline \multicolumn{3}{|c|}{ Tipo de parto } \\
\hline Espontáneo & $441(70,2)$ & $453(68,8)$ \\
\hline Cesárea & $187(29,8)$ & $205(31,2)$ \\
\hline Instrumentado & $0(0,0 \%)$ & $0(0,0 \%)$ \\
\hline \multicolumn{3}{|c|}{ Multiplicidad embarazo } \\
\hline Simple & $626(99,7)$ & $651(98,9)$ \\
\hline Gemelar & $2(0,3)$ & $7(1,1)$ \\
\hline Múltiple & $0(0,0)$ & $0(0,0)$ \\
\hline \multicolumn{3}{|c|}{ Tiempo de gestación } \\
\hline$<37$ semanas & $27(4,3)$ & $36(5,5)$ \\
\hline 37 a 41 semanas & $600(95,5)$ & $620(94,2)$ \\
\hline 42 y más & $1(0,2)$ & $2(0,3)$ \\
\hline \multicolumn{3}{|c|}{ Peso al nacer } \\
\hline$<2500 \mathrm{~g}$ & $26(4,1)$ & $28(4,3)$ \\
\hline$>=2500-4000 \mathrm{~g}$ & $582(92,7)$ & $604(91,8)$ \\
\hline$>4000 \mathrm{~g}$ & $20(3,2)$ & $26(4,0)$ \\
\hline \multicolumn{3}{|c|}{ Sexo del recién nacido } \\
\hline Femenino & $307(48,9)$ & $324(49,2)$ \\
\hline Masculino & $321(51,1)$ & $334(50,8)$ \\
\hline
\end{tabular}

prenatales, y un $7 \%$ tuvo el primer contacto con el servicio de salud en el momento del parto. Un 20\% reportó haber tenido ya más de un embarazo, y un $25 \%$ de ellas un periodo intergenésico menor de dos años. Casi la totalidad tuvo un parto a término, con un peso adecuado para la edad gestacional; de hecho, el bajo peso al nacer es inferior a lo informado en otras estadísticas colombianas o en países como Cuba (20-22). El porcentaje de parto por cesárea fue cercano al $30 \%$ y a partir de las variables de estudio no se encontró morbilidad materna o neonatal importante.

La prevalencia fue ligeramente superior a la reportada para el departamento de Casanare por la Encuesta Nacional de Salud (ENDS-2010) de 20,7\% (10), y a la del país, de 19,5\% (12), pero al comparar con la prevalencia estimada por el DANE para el municipio en 2012, de 22,0\%, no registra cambios (13). La prevalencia nuestra es menor a la informada en Argentina, con un 26\%, y superior a la de Guatemala, con 16\% (19).

En cuanto a la edad promedio, nuestros datos son inferiores a los registrados para la ciudad de Bucaramanga (23), y la proporción de adolescentes menores es similar a la reportada en otros estudios en Bogotá y Cartagena (Colombia) (17-19). La edad del padre mostró una diferencia de aproximadamente 7 años respecto a la de la madre adolescente, hallazgo similar al reportado en el municipio de Nocaima, Cundinamarca (24); la proporción de padres mayores de 20 años es superior a la del país en 11 puntos porcentuales (25). Siete de cada 10 adolescentes embarazadas vive en unión libre, lo cual puede ser determinante en la exposición al riesgo del embarazo (26), y 8 de cada 10 residen en la zona urbana municipal, similar a lo reportado en otros estudios en el país $(23,27)$.

Respecto a la asistencia de al menos a 4 controles prenatales nuestra cifra es similar a la descrita por Althabe para Latinoamérica, del $51 \%$, y mayor a la informada por Silva en Chapoecó, Brasil, entre el 2011-2103, que muestra que el 20\% asistió a menos de 6 controles durante el embarazo $(6,28)$, y menor a la informada por Fajardo en Honduras que informa que el $80 \%$ de las adolescentes no asistieron a control prenatal (29). 
En cuanto a la proporción de gestantes con más de una gestación, nuestros hallazgos son similares a los descritos por Ryan en Perú, con el $18 \%$ (30), y menores al $25 \%$ en Latinoamérica (6); en cuanto al periodo intergenésico, nuestro datos son inferiores a los reportados por González en Chile, que informa que el $36 \%$ de las adolescentes tuvó un periodo integenésico menor a 1 año (31). En cuanto a la proporción de cesáreas esta fue superior al 18,0\% reportado por Amaya et al. en Bogotá (17), y a lo recomendado por la OMS de 10 a $15 \%$ (32), e inferior al informado por Silva en Brasil con el $67 \%$ en el área rural (28).

Como debilidades se evidenciaron fallas en el diligenciamiento del certificado de nacido vivo reportado por las entidades encargadas de la atención del parto a través del sistema RUAF-ND por lo cual el análisis no se hizo con el 100\% de la información suministrada; no obstante, nos permitió una aproximación al análisis de la situación del embarazo adolescente en el municipio de Yopal en el periodo de estudio.

\section{CONCLUSIONES}

La prevalencia del embarazo en adolescentes es alta, por esta razón es importante promover la adherencia al control prenatal en las adolescentes gestantes. Los programas de promoción y prevención que se diseñen para las adolescentes deben tener en cuenta variables como nivel de escolaridad, lugar de residencia y, en general aquellas, condiciones particulares donde se desarrolla y transcurre la vida de esta población. Se requiere continuar la medición de la prevalencia para evaluar el impacto de la estrategia a mediano y largo plazo, y estudios orientados determinar los factores de riesgo de la embarazadas adolecente para intervenir en aquellos factores modificables.

\section{AGRADECIMIENTOS}

A los amigos y excompañeros de aula, y, de manera especial, a la Secretaría de Salud de Yopal cuyo aporte ayudó a documentar y enriquecer esta investigación.

\section{REFERENCIAS}

1. Fondo de las Naciones Unidas para la Infancia (Unicef). Estado mundial de la infancia 2011. La adolescencia una época de oportunidades. Nueva York: Unicef; 2011.

2. Born D, Minujín A, Lombardía ML. Una aproximación a la situación de adolescentes y jóvenes en américa latina y el caribe a partir de evidencia cuantitativa reciente. Panamá: Unicef; 2015. p. 50.

3. Barbón O. Algunas consideraciones sobre comunicación, género y prevención del embarazo adolescente. Rev Cubana Hig Epidemiol. 2012;50:245-9.

4. Organización Mundial de la Salud (OMS). Directrices de la OMS para la prevención del embarazo precoz y los resultados reproductivos adversos en adolescentes de los países en desarrollo. Estados Unidos de América: OMS; 2012.

5. Zamora-Lares AN, Panduro-Barón JG, QuezadaFigueroa NA, González-Moreno J, Fajardo-Dueñas S. Embarazo en adolescentes y sus complicaciones materno perinatales. Rev Med MD. 2013;4:233-8.

6. Althabe F, Moore JL, Gibbons L, Berrueta M, Goudar SS, Chomba E, et al. Adverse maternal and perinatal outcomes in adolescent pregnancies: The Global Network's Maternal Newborn Health Registry study. Reproductive Health. 2015;12:S8. https://doi. org/10.1186/1742-4755-12-S2-S8

7. Organización Mundial de la Salud. El embarazo en la adolescencia 2014 [Visitado 2016 Jul 13]. Disponible en: http:/www.who.int/mediacentre/factsheets/fs364/es/.

8. Organización Mundial de la Salud. Estadísticas sanitarias mundiales 2013. Switzerland: WHO; 2013.

9. Fondo de Población de las Naciones Unidas (UNFPA). Maternidad en la niñez. Enfrentar el reto del embarazo en adolescentes. New York: UNFPA; 2013.

10. Fondo de Población de las Naciones Unidas (UNFPA). La reproducción en la adolescencia y sus desigualdades en América Latina: Informe de la División de Población. Santiago de Chile: Comisión Económica para América Latina y el Caribe (Cepal); 2014.

11. Álvarez VH. Distribución territorial y determinantes de la fecundidad adolescente en Colombia. Notas de población. 2015;101:79-107. 
12. Profamilia, Ministerio de la Protección Social, Instituto Colombiano de Bienestar Familiar (ICBF), Agencia de los Estados Unidos para el Desarrollo Internacional (Usaid). Encuesta Nacional de Demografía y Salud ENDS 2010. Bogotá: Profamilia; 2011.

13. Departamento Administrativo Nacional de Estadística (DANE). Informe de seguimiento de los Objetivos de Desarrollo del Milenio. Bogotá: DANE; 2013.

14. Ministerio de Salud y Protección Social. Política Nacional de Sexualidad, Derechos Sexuales y Derechos Reproductivos. Bogotá: MinSalud; 2014.

15. Ministerio de Salud y Protección Social. Plan Decenal de Salud Pública 2012-2021: La salud en Colombia la construyes tú. Bogotá: MinSalud; 2013.

16. Colombia, Consejo Nacional de Política Económica y Social (Conpes), Departamento Nacional de Planeación (DNP). Lineamientos para el desarrollo de una estrategia para la prevención del embarazo en la adolescencia y la promoción de proyectos de vida para los niños, niñas, adolescentes y jóvenes en edades entre 6 y 19 años. Documento Conpes Social 147 de 2012 (enero 31).

17. Amaya J, Borrero C, Ucrós S. Estudio analítico del resultado del embarazo en adolescentes y mujeres de 20 a 29 años en Bogotá, Colombia. Rev Colomb Obstet Ginecol. 2005;56:216-24.

18. Arrieta JA, Ramos EC, Murillo MA, Mercado KL, Silgado $\mathrm{O}$, Velásquez K, et al. Prevalencia de embarazos en adolescentes escolares en la ciudad de Cartagena. Febrero a junio 2010. Rev Cienc Biomédicas. 2010;1:162-7.

19. Segura O. ¿Fracasaron la consejería preconcepcional y el control prenatal? Una mirada desde las estadísticas vitales en Bogotá (Colombia), 2009-2010. Investig Andina. 2011;13:139-49.

20. Departamento Administrativo Nacional de Estadística (DANE). Nacimientos 2010. Cuadro 7. Nacimientos por grupo de edad de la madre según departamento y municipio de residencia de la madre 2012 [visitado 2015 Feb 2]. Disponible en: http://www.dane.gov.co/ index.php/esp/poblacion-y-demografia/nacimientosy-defunciones/1 18-demograficas/estadisticas-vitales/ 2874-nacimientos-2010-preliminar
21. Instituto Nacional de Salud. Protocolo de Vigilancia en Salud Pública de Bajo peso al nacer a término. Bogotá: INS; 2014.

22. Rodríguez-Pérez M, Mora-Rodríguez M, Mora-Vera I. Embarazo en la adolescencia y su relación con el recién nacido con bajo peso. Rev Cubana Enfermer. 2001;17:101-6.

23. Blanco-Gómez A, Osma-Zambrano S, MonturiolDurán J, Medina-Pabón M, Quintero-Díaz LM, Alfonso-Hernández EP. Prevalencia de partos en la adolescencia y factores asociados en el Hospital Universitario de Santander, Bucaramanga (Colombia) 2008. Estudio de corte transversal. Rev Colomb Obstet Ginecol. 2010;61:113-20.

24. Alvarado R, Martínez OA, Baquero M, Valencia CM, Ruiz AMV. Problemática en torno al embarazo en adolescentes de 14 a 19 años en Nocaima (Cundinamarca). Rev Cienc Salud. 2007;5:40-52.

25. Vega GA. Análisis de la línea base del Observatorio de Embarazo Adolescente en Colombia: Ministerio de Salud y Protección Social. Observatorio Nacional Intersectorial de embarazo en la Adolescencia; 2013.

26. Flórez CE, Soto VE. Factores protectores y de riesgo del embarazo adolescente en Colombia. Estudio a profundidad basado en las Encuestas Nacionales de Demografía y Salud (ENDS-1990/2010). Bogotá: Profamilia; 2013.

27. Contreras JB. Prevalencia de embarazo en adolescentes en la ESE Alejandro Próspero Reverendo de Santa Marta en los años 2008-2009. Bogotá: Universidad Nacional de Colombia; 2011.

28. Silva RA, Ferraz L, Busato MA. Perfil epidemiológico dos nascidos vivos no município de Chapecó-SC. RECIIS - Rev Eletron Comun Inf Inov Saúde. 2016;10:1-16.

29. Fajardo Hernández IJ, Ramos Escalantes GJ, Padilla Maldona JA, Andrade Sánchez, SC, Cuadra Morales YM . Prevalencia de embarazo de adolescentes, Hospital Leonardo Martínez Valenzuela. Rev Cient Esc Univ Cienc Salud. 2015;2:10-20.

30. Ryan JA, Casapía M, Aguilar E, Silva H, Joseph SA, Gyorkos TW. Comparison of prenatal care coverage 
in early adolescents, late adolescents, and adult pregnant women in the Peruvian Amazon. Int J Gynaecol Obstet. 2009;107:162-5. doi: 10.1016/j. ijgo.2009.06.01.

31. González AE. Embarazo repetido en la adolescencia: la realidad chilena. Rev Chil Obstet Ginecol. 2016; 81:354-9.
32. Organización Mundial de la Salud. Solo se deben practicar las cesáreas que sean necesarias por motivos médicos 2015 [visitado 2017 Ene 17]. Disponible en: http:/www.who.int/mediacentre/news/releases/2015/ caesarean-sections/es/.

\section{Conflicto de intereses: ninguno declarado.}

